EPJ Web of Conferences 113,05011 (2016)

DOI: $10.1051 /$ epjconf/201611305011

(C) Owned by the authors, published by EDP Sciences, 2016

\title{
Quarks in Few Body Nuclei
}

\author{
Roy J. Holt ${ }^{1, a}$ \\ ${ }^{1}$ Physics Division, Argonne National Laboratory, Argonne, Illinois 60439
}

\begin{abstract}
Electron scattering at very high Bjorken $x$ from hadrons provides an excellent test of models, has an important role in high energy physics, and from nuclei, provides a window into short range correlations. Light nuclei have a key role because of the relatively well-known nuclear structure. The development of a novel tritium target for Jefferson Lab has led to renewed interest in the mass three system. For example, deep inelastic scattering experiments in the light nuclei provide a powerful means to determine the neutron structure function. The isospin dependence of electron scattering from mass3 nuclei provide information on short range correlations in nuclei. The program using the new tritium target will be presented along with a summary of other experiments aimed at revealing the large-x structure of the nucleon.
\end{abstract}

\section{Introduction}

During the past decades there has been an enormous effort to determine the parton distribution functions (PDFs) of the most stable hadrons: neutron, proton and pion [1]. The behavior of the PDFs on the far valence region (Bjorken- $x>0.5$ ) is of particular interest because this domain defines a hadron. Recognizing the importance of the far valence region, a new generation [2] of experiments, focused on $x \gtrsim 0.5$, is planned at the Thomas Jefferson National Accelerator Facility (JLab), and under examination in connection with Drell-Yan studies at the Fermi National Accelerator Facility (FNAL) [3] and a possible Electron Ion Collider (EIC) [4]. One of the new experiments required the development of a tritium target for JLab, which led to new planned experiments. Theoretical calculations [5-12] of the pion structure function have demonstrated the success of the nonperturbative approach in QCD. Finally, the high- $x$ region could impact high energy physics since low momentum transfer and high $x$ evolves to high momentum transfer and low $x$. This talk will focus on three main areas of research at JLab: first, the planned measurements of the $F_{2}^{n} / F_{2}^{p}$ and $d / u$ ratios as well as present status; secondly, measurements of the longitudinal spin asymmetries for the proton and neutron, and third, the planned measurements that will make use of the new low-activity tritium target developed for JLab.

\section{Status of high-x experiments}

Two types of experiments will be extremely useful for understanding models of the nucleon. First, measurements of the $F_{2}^{n} / F_{2}^{p}$ ratio at very high $x$ are essential to constrain the models. Secondly, accurate, high-x, polarized, longitudinal asymmetries for both the proton and neutron are extremely important to understand the nucleon structure.

\footnotetext{
a e-mail: holt@anl.gov
} 


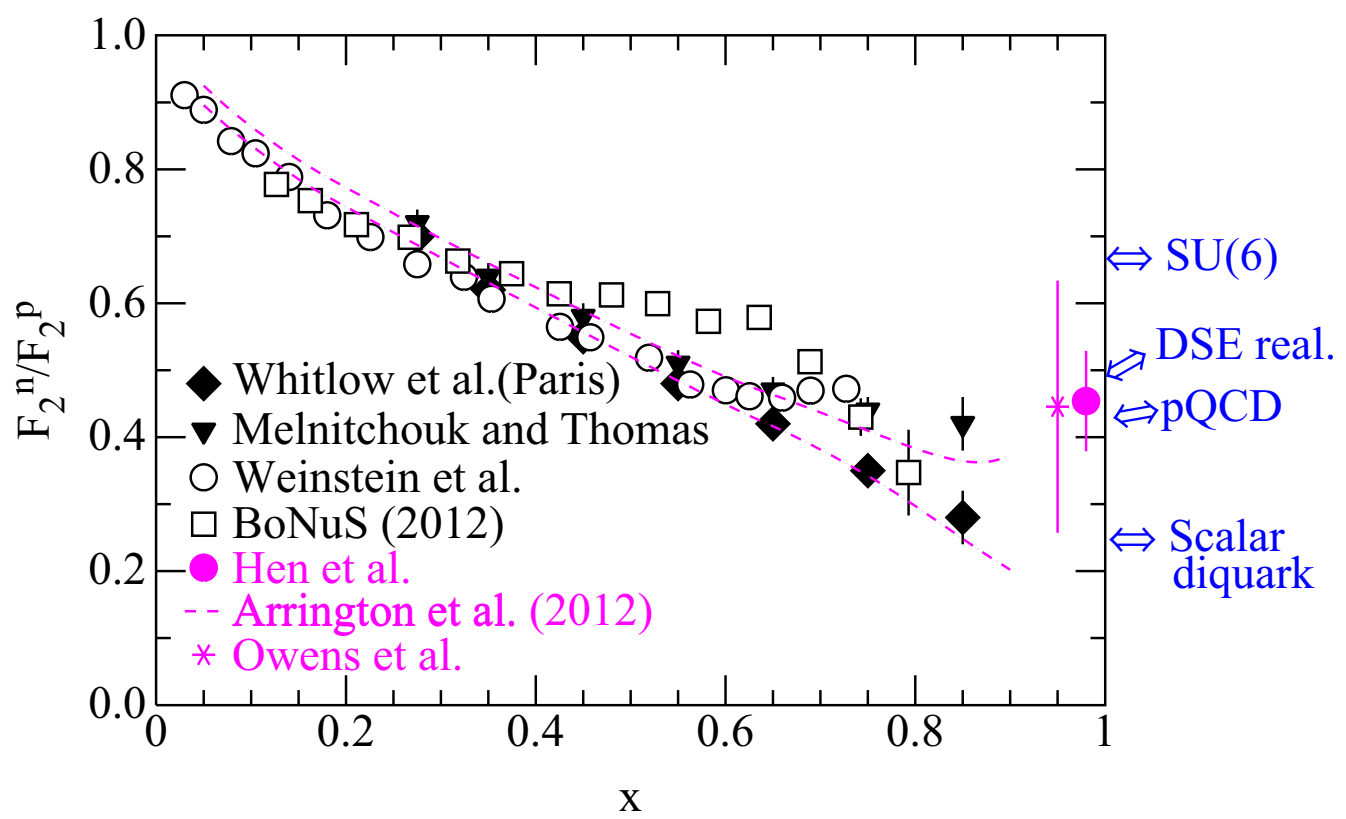

Figure 1. $F_{2}^{n} / F_{2}^{p}$ as a function of $x$. Results from various extraction methods are shown [14-21] along with selected predictions. Adapted from ref. [5].

\section{$2.1 F_{2}^{n} / F_{2}^{p}$ ratio at high $x$}

There is considerable theoretical interest in the ratio of $F_{2}^{n} / F_{2}^{p}$ and $d / u$ at very high $x$. The ratio as $x \rightarrow 1$ is sensitive to the model of the nucleon. One can see from the right side of Fig. 1 that the $F_{2}^{n} / F_{2}^{p}$ or $d / u$ ratio varies substantially among the models. Within the parton model at very high x:

$$
\frac{F_{2}^{n}}{F_{2}^{p}} \stackrel{x \simeq 1}{=} \frac{1+4\left(d_{v} / u_{v}\right)}{4+\left(d_{v} / u_{v}\right)} .
$$

Thus a measurement of the neutron and proton structure functions at large- $x$ provides a determination of the $d_{v} / u_{v}$ ratio. However, while proton and deuteron DIS data are well measured at reasonably high $x$, the extraction of the neutron structure function at very high $x$ from DIS data on the deuteron is problematic. The central difficulty is that the extraction of $F_{2}^{n} / F_{2}^{p}$ at high $x$ is sensitive to the poorly known high-momentum components of the deuteron wave function [13].

Many extractions of the neutron-proton structure function ratios have been performed [14-22]. They are summarised in Fig. 1, with recent inferences indicated by the points with error bars near $x=1$ : there is a large uncertainty in the ratio for $x \gtrsim 0.6$. (See also Fig. 25 in Ref. [1].) A very recent work [23] has found that the $F_{2}^{n} / F_{2}^{p}$ ratio rises rapidly at large $x$, and consequently, has opened up the uncertainty range once again. New experimental methods are necessary in order to place tighter constraints on the far valence domain. A primary goal is to empirically eliminate or constrain the quite different theoretical predictions.

Two new experiments $[24,25]$ should provide data up to $x \approx 0.85$ with the upgraded beam energy at JLab. Since much of the uncertainty can be traced to the poorly known short-range part of 
the deuteron wave function, the JLab BoNuS Collaboration has performed [21] and will perform [24] an experiment where a very low energy spectator proton from the deuteron can be detected in coincidence with a DIS event from the neutron in the deuteron. In this way, one can restrict the data to a region where the well-known long-range part of the deuteron wave function dominates the process. The central difficulty with this experiment is detecting the very low energy proton of about 150 $\mathrm{MeV} / \mathrm{c}$ or less. This requires extremely thin target and detector components. An interesting variant of this approach is to use an EIC with, e.g., an $8 \mathrm{GeV}$ electron beam impinging on a deuteron beam of $30 \mathrm{GeV} /$ nucleon. The forward going energetic proton would be detected at very small angles in coincidence with a DIS event from the neutron. Simulations suggest that this should be feasible [26].

Another method is to perform deep inelastic scattering from the mirror nuclei ${ }^{3} \mathrm{He}$ and ${ }^{3} \mathrm{H}$ over a broad range in $x[25,27-30]$. Theoretical calculations indicate that nuclear effects cancel to a high degree in extracting the $F_{2}^{n} / F_{2}^{p}$ ratio from these two nuclei. This experiment would also be useful in determining the EMC effect in the mass-three system [31]. Although providing a tritium target for $\mathrm{JLab}$ is straightforward [32], it is not trivial. The necessary tritium target has been developed for JLab and will be installed in 2016.

Finally, parity violating DIS can avoid the problem encountered with neutrons bound in nuclei. Parity-violating DIS from the proton is sensitive to the $d / u$ ratio [33]. The proposed SoLID experiment plans to measure the $d / u$ ratio up to an $x$ of 0.7 .

\subsection{Longitudinally polarized deep inelastic scattering at high $x$}

It is evident from the right side of Fig. 2 that measurements of the longitudinal asymmetries in DIS provide an important constraint on models of nucleon structure. Numerous experiments and extractions aimed at determining nucleon longitudinal spin structure functions have been performed [34].

Existing measurements of $A_{1}^{p}$ are summarised in Fig. 2. Unfortunately, these data are not of sufficient accuracy and high enough $x$ to discriminate among the models. As indicated in Fig. 2, however, a new JLab experiment [35] will extend the results up to $x \approx 0.8$ with a projected error that promises a significant constraint on the models.

The status of existing data for $A_{1}^{n}$ is shown for polarized ${ }^{3} \mathrm{He}$ targets in Fig. 3. The data extend only to $x \approx 0.6$ and place little constraint on descriptions of the nucleon. New experiments proposed at $\mathrm{JLab}[36,37]$ are expected to provide results up to $x \approx 0.75$, as indicated in Fig. 3. These new results should permit discrimination between the $\mathrm{pQCD}$ model and other predictions.

\section{New studies in the mass- 3 system}

Three other experiments that make use of the new tritium target at JLab are: (i) $x>1$ measurements [38], (ii) nucleon momentum distributions in three body nuclei [39] and (iii) the ${ }^{3} \mathrm{H}-{ }^{3} \mathrm{He}$ charge radius difference measurement [40]. It is well established [41] that $x>1$ experiments are sensitive to shortrange nucleon-nucleon correlations (SRC) in nuclei. An issue is the isospin dependence of the SRC. The ${ }^{3} \mathrm{He}-{ }^{3} \mathrm{H}$ system represents the best opportunity to observe isospin differences since ${ }^{3} \mathrm{He}$ has the largest $\mathrm{Z} / \mathrm{N}$ ratio available and ${ }^{3} \mathrm{H}$ has a $2: 1 \mathrm{~N} / \mathrm{Z}$ ratio. In fact, a simple estimate gives the ratio of ${ }^{3} \mathrm{He}$ to ${ }^{3} \mathrm{H} x>1$ cross section to be a factor of 1.4 ; whereas, for complete $\mathrm{n}-\mathrm{p}$ dominance of the SRC, the ratio is expected to be unity. This experiment would be expected to extract the ratio of the $T=1$ to $T=0$ component to better than $4 \%$.

An especially clever way to measure the ratio of the neutron to proton momentum distributions in nuclei is to use the mirror nuclei, ${ }^{3} \mathrm{H}$ and ${ }^{3} \mathrm{He}$, and measure only (e,e'p) reactions for both nuclei [39]. This avoids the technically difficult problem of using only ${ }^{3} \mathrm{He}$, say, and detecting both (e,e'n) 


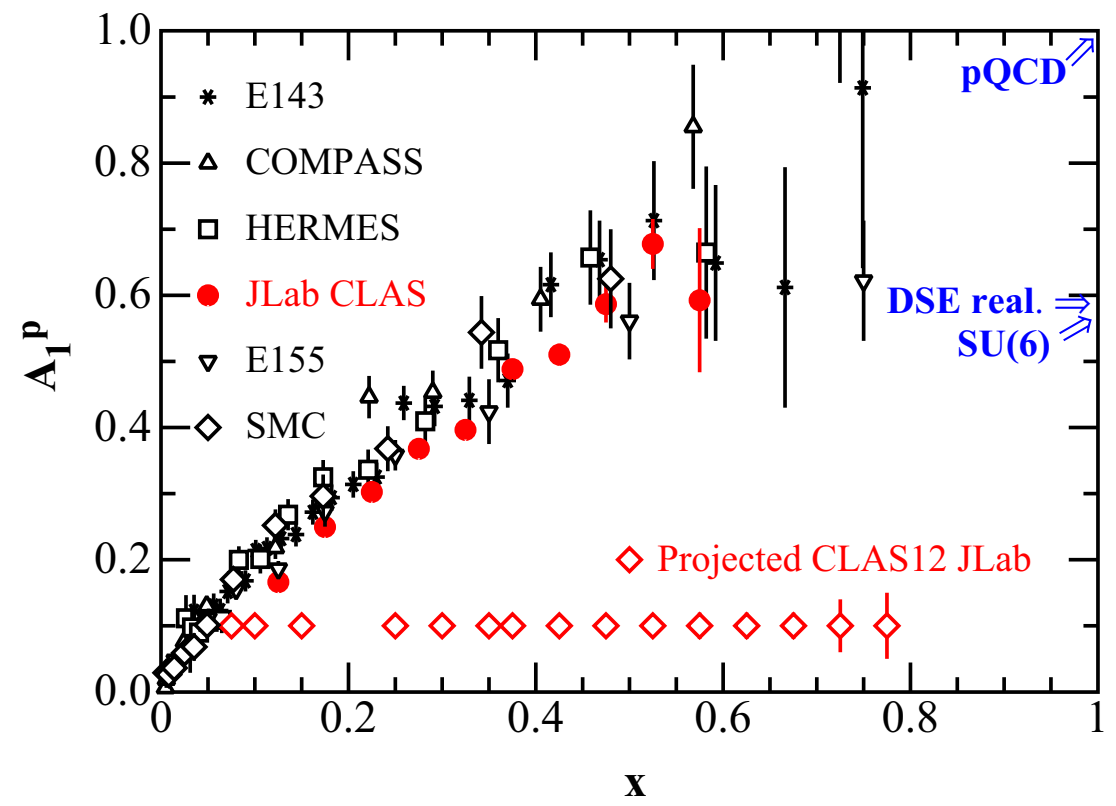

Figure 2. Existing and projected measurements of the proton's longitudinal spin asymmetry as a function of $x$ (statistical errors only), along with selected predictions. Adapted from ref. [5].

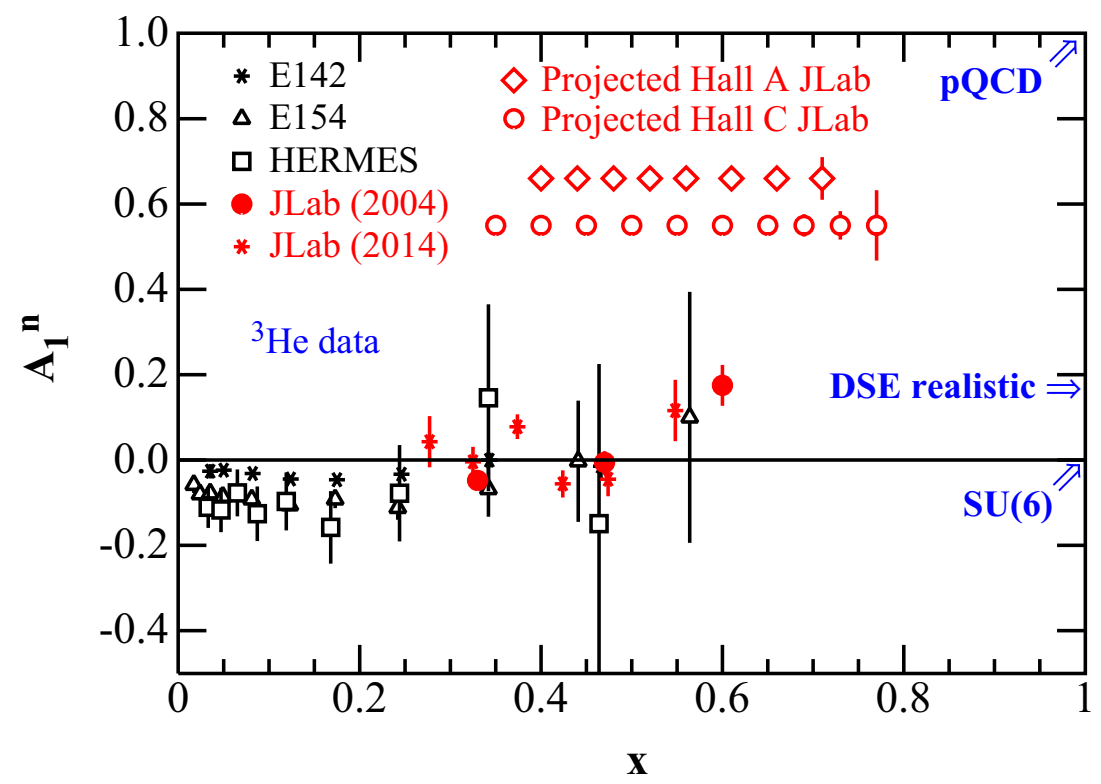

Figure 3. Existing and projected measurements of the neutron's longitudinal spin asymmetry as a function of $x$ (statistical errors only), along with selected predictions. Here, only $A_{1}^{n}$ data obtained from polarised ${ }^{3} \mathrm{He}$ targets are displayed. Adapted from ref. [5]. 
and (e,e'p) reactions. This experiment is especially sensitive to the N-N interaction in the nuclear medium. For example, under total n-p dominance of the N-N interaction, the ratio should be unity. This experiment is tentatively scheduled to run in conjunction with the other tritium experiments at JLab.

The third tritium experiment [40]is aimed at measuring the electric form factor ratio for $3 \mathrm{He}$ and $3 \mathrm{H}$ at low momentum transfer. The goal is to provide an accurate difference between the charge radii for these mirror nuclei.

\section{Summary}

Two and three-body systems have a key role in determining the quark structure of the nucleon. JLab at $12 \mathrm{GeV}$ is an ideal facility for beginning the study of the far valence region of the nucleon, and the EIC should provide a large dynamic range in $Q^{2}$ and $W$ for these studies. The advent of a tritium target at JLab presents new physics opportunities. These new experiments should provide benchmark data for the studies of the nucleon structure, the short range N-N interaction and the structure of 3body nuclei. Possible experiments that would make use of a polarized tritium target at JLab should be evaluated.

\section{Acknowledgements}

I am grateful to C. D. Roberts and J. Arrington for fruitful discussions. This work was funded by the Department of Energy, Office of Science, Office of Nuclear Physics, contract no. DE-AC02-06CH11357.

\section{References}

[1] R.J. Holt, C.D. Roberts, Rev. Mod. Phys. 82, 2991 (2010)

[2] R.J. Holt (2013), arXiv: 1311. 1527

[3] P. Reimer, D. Geesaman et al. (Fermilab E906/Drell-Yan), E906: Fermilab Proposal 906, 2006

[4] A. Accardi, J. Albacete, M. Anselmino, N. Armesto, E. Aschenauer et al. (2012), 1212 . 1701

[5] C.D. Roberts, R.J. Holt, S.M. Schmidt, Phys.Lett. B727, 249 (2013), 1308 . 1236

[6] L. Chang et al., Phys. Rev. Lett. 110, 132001 (2013)

[7] I. Cloët, L. Chang, C. Roberts, S. Schmidt, P. Tandy, Phys.Rev.Lett. 111, 092001 (2013), 1306.2645

[8] L. Chang, I. Cloët, C. Roberts, S. Schmidt, P. Tandy, Phys.Rev.Lett. 111, 141802 (2013), 1307.0026

[9] M.B. Hecht, C.D. Roberts, S.M. Schmidt, Phys. Rev. C 63, 025213 (2001)

[10] K. Wijesooriya, P.E. Reimer, R.J. Holt, Phys. Rev. C 72, 065203 (2005)

[11] M. Aicher, A. Schafer, W. Vogelsang, Phys.Rev.Lett. 105, 252003 (2010), 1009. 2481

[12] T. Nguyen, A. Bashir, C.D. Roberts, P.C. Tandy, Phys. Rev. C 83, 062201(R) (2011)

[13] J. Arrington, D.W. Higinbotham, G. Rosner, M. Sargsian, Prog. Part. Nucl. Phys. 67, 898 (2012)

[14] L. Whitlow, E. Riordan, S. Dasu, S. Rock, A. Bodek, Phys.Lett. B282, 475 (1992)

[15] W. Melnitchouk, A.W. Thomas, Phys.Lett. B377, 11 (1996), nucl-th/9602038

[16] J. Arrington, F. Coester, R.J. Holt, T.S.H. Lee, J.Phys. G36, 025005 (2009), 0805 . 3116

[17] L. Weinstein, E. Piasetzky, D. Higinbotham, J. Gomez, O. Hen et al., Phys.Rev.Lett. 106, 052301 (2011), 1009.5666 
[18] O. Hen, A. Accardi, W. Melnitchouk, E. Piasetzky, Phys. Rev. D 84, 117501 (2011), 1110. 2419

[19] J. Arrington, J. Rubin, W. Melnitchouk, Phys. Rev. Lett. 108, 252001 (2012), 1110. 3362

[20] J. Owens, A. Accardi, W. Melnitchouk, Phys. Rev. D 87, 094012 (2013)

[21] N. Baillie et al. (CLAS Collaboration), Phys. Rev. Lett. 108, 199902 (2012), 1110 . 2770

[22] S. Tkachenko et al. (CLAS), Phys. Rev. C89, 045206 (2014), [Addendum: Phys. Rev.C90,059901(2014)], 1402.2477

[23] W. Cosyn, M.M. Sargsian (2015), 1506.01067

[24] S. Bueltmann et al. (BoNuS Collaboration), The Structure of the Free Neutron at Large $x$ Bjorken, JLab Experiment E12-06-113

[25] G. Petratos et al. (MARATHON Collaboration), Measurement of the $F_{2}^{n} / F_{2}^{p}, d / u$ Ratios and the $A=3$ EMC Effect in Deep Inelastic Scattering off the Tritium and Helium Mirror Nuclei, JLab Experiment E12-06-118

[26] A. Accardi, C. Keppel, R. Ent (2010), Presentation at the "Workshop on Nuclear Chromodynamics with an EIC", Argonne National Laboratory

[27] I.R. Afnan et al., Phys. Lett. B 493, 36 (2000)

[28] F.R.P. Bissey, A.W. Thomas, I.R. Afnan, Phys. Rev. C 64, 024004 (2001)

[29] I.R. Afnan et al., Phys. Rev. C 68, 035201 (2003)

[30] R.J. Holt, G.G. Petratos, AIP Conf.Proc. 1369, 106 (2011)

[31] O. Hen, D. Higinbotham, G. Miller, E. Piasetzky, L. Weinstein, Int. J. Mod. Phys. E. 22, 1330017 (2013)

[32] B. Brajuskovic, T. O’Connor, R. Holt, J. Reneker, D. Meekin et al., Nucl.Instrum.Meth. A729, 469 (2013), 1306.6000

[33] P. Souder et al. (SoLID Collaboration), Precision Measurement of Parity Violation in Deep Inelastic Scattering over a Broad Kinematic Range, JLab Experiment E10-007

[34] C.A. Aidala, S.D. Bass, D. Hasch, G.K. Mallot, Rev.Mod.Phys. 85, 655 (2013), 1209. 2803

[35] S. Khun et al. (CLAS Collaboration), The Longitudinal Spin Structure of the Nucleon, JLab Experiment E12-06-109

[36] X. Zheng et al. (E12-06-110 Collaboration), Measurement of Neutron Spin Asymmetry $A_{1}^{n}$ in the Valence Quark Region using an $11 \mathrm{GeV}$ Beam and a Polarized ${ }^{3}$ He Target in Hall C, JLab Experiment E12-06-110

[37] N. Liyanage et al. (E12-06-122 Collaboration), Measurement of Neutron Spin Asymmetry $A_{1}^{n}$ in the Valence Quark Region using 8.8 and $6.6 \mathrm{GeV}$ Beam Energies and Bigbite Spectrometer in Hall A, JLab Experiment E12-06-122

[38] P. Solvignon et al. (JLab E12-11-112 Collaboration), Jefferson Lab Proposal 12-11-112

[39] O. Hen, L. Weinstein, S. Gilad, W. Boeglin (2014), arXiv: 1410 . 4451

[40] L.S. Myers et al. (JLab E12-14-009 Collaboration), Jefferson Lab Proposal 12-14-009

[41] J. Arrington, A. Daniel, D. Day, N. Fomin, D. Gaskell et al., Phys.Rev. C86, 065204 (2012), 1206.6343 